\title{
Über das Verhalten einiger Polypeptide gegen das Plasma des Rinderblutes.
}

\author{
Von
}

\author{
Emil Abderhalden und James S. McLester, Birmingham. \\ (Aus dem chemischen Institut der Universität Berlin.) \\ (Der Redaktion zugegangen am 17. März 1908.)
}

Die folgenden Untersuchungen schließen sich eng an die entsprechenden Versuche des einen von uns mit $O$ ppler $^{1}$ ) an und sind mit dem Plasma desselben Blutes ausgeführt, das zur Verfolgung des Verhaltens der roten Blutkörperchen und Blutplättchen gegen Polypeptide diente. ${ }^{2}$ ) Das Plasma war durch Zentrifugieren von ganz frischem, durch Zusatz von Ammoniumoxalat ungerinnbar gemachtem Rinderblut gewonnen worden. Das Plasma wurde zunächst durch Zentrifugieren von den roten und weißen Blutkörperchen befreit, dann abgehoben und nun so lange weiter zentrifugiert, bis keine Spur eines Bodensatzes mehr sichtbar war. Das auf diese Weise von allen Zellelementen sorgfältig befreite Plasma wurde nur dann verwendet, wenn keine Spur Hämoglobin nachweisbar war. Wiederholt war das Plasma hämoglobinhaltig und konnte nicht zu unseren Versuchen verwendet werden, denn es kam uns darauf an, zu verhüten, daß aus den roten Blutkörperchen stammende Elemente bei diesen Versuchen vorhanden waren. Ebenso verzichteten wir auf die Benützung des Plasmas und des Blutes überhaupt, wenn Gerinnungen eingetreten waren. Wir hoffen,

1) Emil Abderhalden und Berthold Oppler, Über das Verhalten einiger Polypeptide gegen Blut-Plasma und -Serum vom Pferde, Diese Zeitschrift, Bd. LIII, S. 294, 1907.

2) Vgl. Emil Abderhalden und Wilfred H. Manwaring, Über den Abbau einiger Polypeptide durch die roten Blutkörperchen und die Blutplättchen des Rinderblutes, Diese Zeitschrift, Bd. LV, S. 377, 1908.

Hoppe-Seyler's Zeitschrift f. physiol. Chemie. LV. 
alle Vorsichtsmaßregeln gebraucht zu haben, um alle dem Plasma als solchem nicht zukommenden Elemente auszuschließen. Absolut sicher können wir nicht behaupten, daß nicht doch einzelne weiße und rote Blutkörperchen und Blutplättchen zerfallen und zur Auflösung gekommen sind. Da jedoch in einzelnen Punkten die genannten Elemente und das Plasma sich ganz verschieden gegen bestimmte Polypeptide verhalten haben, sind wir wohl berechtigt, anzunehmen, daß die am Plasma gemachten Beobachtungen auf dieses selbst und nicht auf zufällige Beimengungen zurückzuführen sind. Wir heben diese Schwierigkeiten absichtlich deutlich hervor, um zu zeigen, wie vorsichtig die Beurteilung derartiger Versuche vorgenommen werden muß. Es sind noch sehr viele Untersuchungen nach dieser Richtung mit verschiedenen Blutarten und unter verschiedenen Bedingungen erforderlich, ehe ein bestimmtes abschließendes Urteil über den Wert dieser Beobachtungen möglich sein wird. Wir teilen aus diesem Grunde vorläufig nur die gefundenen Tatsachen mit.

Der Gang der Untersuchung war in den Grundzügen genau derselbe, wie er in der erwähnten Arbeit ${ }^{1}$ ) geschildert worden ist. Die $z u$ den Versuchen bestimmten Polypeptide wurden in einer abgemessenen Menge Plasma gelöst und nach Zusatz von Toluol die Lösung 4 Tage bei $37^{\circ}$ aufbewahrt. Die Entfernung des Eiweißes nahmen wir durch Kochen mit Tierkohle oder durch Schütteln mit Kaolin vor. ${ }^{2}$ ) Das eiweißfreie Filtrat wurde unter vermindertem Druck bei $40^{\circ}$ des Wasserbades zur Trockene verdampft. Den Rückstand veresterten wir in gewohnter Weise und mit den üblichen Vorsichtsmaßregeln. Die Ester wurden mit der auf den Chlorgehalt berechneten Menge Natriumalkoholat in Freiheit gesetzt und dann bis $100^{\circ}$ des Wasserbades bei einem Druck von 10-12 mm destilliert. Das Destillat wurde unter sehr guter Kühlung aufgefangen und sofort unter Zusatz von wässeriger Salzsäure in einer gewogenen Schale zur Trockene verdampft. Der Rückstand wurde über Kalk und Schwefelsäure getrocknet und dann gewogen.

1) 1. c.

2) P. Rona und L. Michaelis, Weitere Beiträge zur Methodik der Enteiweißung, Biochem. Zeitschrift, Bd. V, S. 365, 1907. 
War Glykokoll zu erwarten, so wurden die salzsauren Aminosäuren mit Alkohol übergossen und gasförmige, trockene Salzsäure eingeleitet. Durch Impfen eines Kryställchens von Glykokollesterchlorhydrat und Abkühlen wurde dann das Glykokoll als salzsaurer Ester zur Abscheidung gebracht. Die Mutterlauge der Abscheidung dampften wir wieder mit wässeriger Salzsäure zur Trockene ein, bestimmten das Gewicht des Rückstandes und stellten sein spezifisches Drehungsvermögen fest. Dieser Gang der Untersuchung des Destillates wurde bei der Verwendung von Glycyl-dl-alanin und dl-Alanyl-glycin befolgt. Hier handelte es sich um den Nachweis von Glykokoll und d-Alanin. Bei Verwendung von Diglycyl-glycin und von Glycyl-l-tyrosin kam im Destillat nur Glykokoll in Betracht. Das 1-Tyrosin suchten wir bei Anwendung von Glycyl-l-tyrosin durch direkte Krystallisation aus der enteiweißten Flüssigkeit zu gewinnen. In allen Fällen wurde der Destillationsrückstand in Alkohol gelöst und der Dipeptidester in das entsprechende Anhydrid übergeführt. Zur Identifizierung diente der Schmelzpunkt, die Feststellung, daß ein Anhydrid vorlag (kein Kupfersalz beim Kochen der wässerigen Lösung mit frisch gefälltem Kupferoxyd), und endlich prüften wir bei Anwendung von Glycyl-dl-alanin und dl-Alanyl-glycin das optische Verhalten des isolierten Anhydrids. Wir heben nochmals hervor, daß die racemischen Polypeptide für alle derartigen Versuche von größtem Werte sind, indem sie gestatten, sofort festzustellen, ob das erhaltene Resultat - angenommen, es sei eine Hydrolyse beobachtet worden - auf eine fermentative Spaltung zurückgeführt werden darf oder aber der angewandten Methode zur Last fällt. Ist die Spaltung asymmetrisch verlaufen, dann ist bewiesen, daß ein Fermentprozeß vorliegt, sind dagegen die Spaltprodukte inaktiv; dann liegt der Einwand nahe, daß Fehler bei der Durchführung der Methode vorgekommen sind.

Aus den unten mitgeteilten Beobachtungen ergibt sich, daß das Plasma des untersuchten Rinderblutes Gl y cyl-l-ty rosin zum Teil gar nicht gespalten hat, zum Teil in geringfügiger Weise. Eine umfangreichere Spaltung fand in keinem Falle statt. Es ist dieses Resultat deshalb von Interesse, weil die 
roten Blutkörperchen derselben Blutportion Glycyl-l-tyrosin rasch und in großem Umfange spalteten. Auch hämoglobinhaltiges Plasma zeigte eine deutliche Hydrolyse von Glycyl-l-tyrosin. Glycyl-dl-alanin und dl-Alanyl-glycin wurden gespalten und ebenso Diglycyl-glycin. Das erstere Dipeptid wurde allerdings stets in nur geringer Menge hydrolysiert, während bei dl-Alanyl-glycin und Diglycyl-glycin die Spaltung eine recht beträchtliche war. Dieses Resultat stimmt mit den beim Plasma des Pferdeblutes gemachten Beobachtungen gut überein, nur wurde bei diesem Glycyl-dl-alanin zum Teil überhaupt nicht in nachweisbarer Menge angegriffen.

\section{Experimenteller Teil.}

Serie I.

1. Glycyl-1-tyrosin: $1 \mathrm{~g}$ in $25 \mathrm{ccm}$ Plasma gelöst, nach Zusatz von Toluol 4 Tage in Brutraum aufbewahrt. Die Lösung zeigte einen ziemlich beträchtlichen Bodensatz, dessen Untersuchung ergab, daß Eiweiß vorlag. Tyrosin war nicht zur Ausscheidung gelangt. Es glückte nicht, l-Tyrosin aus der enteiweißten Flüssigkeit durch Krystallisation zu erhalten. Auch Glykokoll konnte nicht als Esterchlorhydrat nachgewiesen werden. Wie das aus dem bei der Destillation verbleibenden Rückstand gewonnene Glycyl-l-tyrosinanhydrid ergab, war eine irgendwie beträchtliche Spaltung des Glycyl-l-tyrosins nicht erfolgt. Eis wurden $0,7 \mathrm{~g}$ reines Glycyl-l-tyrosinanhydrid erhalten.

2. dl-Alanyl-glycin: $2 \mathrm{~g}$ in $25 \mathrm{ccm}$ Plasma gelöst. 4 Tagıe im Brutraum aufbewahrt unter Zusatz von Toluol.

Isoliert: 0,21 g Glykokollesterchlorhydrat (F. $144^{\circ}$ [korr.])), $0,20 \mathrm{~g}$ d-Alanin $\left([\alpha]_{\mathrm{D}}^{200}\right.$ des salzsauren Salzes $\left.+8,0^{\circ}\right), 0,40 \mathrm{~g}$ Glycyl:l-alaninanhydrid $\left([\alpha]_{\mathrm{D}}^{20^{0}}=+4,5^{0}\right.$ in wässeriger Lösung; F. $\left.240^{\circ}\right)$ ), 0,75 g Anhydrid vom F. $235^{\circ}$, optisch fast inaktiv, nicht ganz rein. 3. Glycyl-dl-alanin: $2 \mathrm{~g}$ in $25 \mathrm{ccm}$ Plasma gelöst. Nach 4 tägigem Stehen bei $37^{\circ}$ verarbeitet. Gewonnen 0,12 Glykokollesterchlorhydrat (F. $144^{\circ}$ [korr.]) und 0,10 g d-Alanim (das salzsaure Salz zeigte $[\alpha]_{D}^{20^{0}}=+7,5^{0}$ ). Aus dem De stillationsrückstand wurden erhalten $0,3 \mathrm{~g}$ Glycyl-1-alaninan:- 
hydrid: $[\alpha]_{\mathrm{D}}^{20^{\circ}}=+3,2^{\circ}$ in wässeriger Lösung und ferner 3 Fraktionen von zusammen $1,0 \mathrm{~g}$ Anhydrid vom F. $=240^{\circ}$. Alle drei Fraktionen waren optisch inaktiv.

4. Diglycyl-glycin: $\mathbf{2} \mathrm{g}$ in $25 \mathrm{ccm}$ Plasma gelöst. 4 Tage im Brutraum aufbewahrt. Isoliert $1,0 \mathrm{~g}$ Glykokollesterchlorhydrat (F. $144^{\circ}$ korr.) und 0,50 g Glycinanhydrid (F. unter Zersetzung gegen $305^{0}$ [korr.]).

\section{Serie II.}

Alle Polypeptide wurden in je $25 \mathrm{ccm}$ Plasma gelöst und verblieben 4 Tage im Brutraum.

1. Glycyl-l-tyrosin: Angewandt $1 \mathrm{~g}$. Kein Tyrosin isoliert und nur Spuren von Glykokollesterchlorhydrat. $0,58 \mathrm{~g}$ Glycyl-l-tyrosinanhydrid.

2. dl-Alanyl-glycin: Angewandt $2 \mathrm{~g}$. Isoliert: $0,42 \mathrm{~g}$ Glykokollesterchlorhydrat (F. $144^{\circ}$ [korr.]), 0,30 g d-Alanin $\left([\alpha]_{D}^{200}\right.$ des salzsauren Salzes $\left.=+9,8^{\circ}\right)$. Aus dem Destillationsrückstand gewannen wir $0,25 \mathrm{~g}$ Glycyl-l-alaninanhydrid. Es zeigte in wässeriger Lösung $[\alpha]_{\mathrm{D}}^{200}=+3,5^{\circ}$. Eine zweite Fraktion von $0,15 \mathrm{~g}$ drehte $+2,5^{\circ}$ und eine dritte $0,25 \mathrm{~g}$ wiegende Fraktion war optisch inaktiv.

3. Glycyl-dl-alanin: Verwendet $2 \mathrm{~g}$. Isoliert: $0,15 \mathrm{~g}$ Glykokollesterchlorhydrat und 0,10 g d-Alanin. Letzteres war sehr unrein und zeigte als salzsaures Salz nur $[\alpha]_{\mathrm{D}}^{200^{\circ}}=+6,0^{\circ}$. Optisch aktives Anhydrid konnte in reinem Zustand nicht gewonnen werden. Die Gesamtausbeute an Anhydrid betrug 1,2 g. F. $240^{\circ}$.

4. Diglycyl-glycin: Angewandt $2 \mathrm{~g}$. Isoliert: $0,68 \mathrm{~g}$ Glykokollesterchlorhydrat. F. $140^{\circ}$ (korr.). Aus dem Destillationsrückstand erhielten wir $0,70 \mathrm{~g}$ reines Glycinanhydrid (Zersetzungspunkt $305^{\circ}$ (korr.).

Serie III.

Alle Proben verblieben 4 Tage im Brutraum.

1. Glycyl-l-tyrosin: $1 \mathrm{~g}$ angewandt und in $25 \mathrm{ccm}$ Plasma gelöst. Isoliert: 0,05 g l-Tyrosin und 0,08 g Glykokoll- 
esterchlorhydrat. F. $144^{\circ}$ (korr.) An Anhydrid wurden zurückgewonnen $0,65 \mathrm{~g}$.

2. Glycyl-l-t yrosin: $1 \mathrm{~g}$ verwendet und in $25 \mathrm{ccm}$ Plasma gelöst. 0,06 g l-Tyrosin isoliert und 0,075 g Glykokollesterchlorhydrat (F. $144^{\circ}$ [korr.]). Zurückgewonnen 0,50 g Anhyydrid.

3. dl-Alanyl-glycin: $2 \mathrm{~g}$ in $25 \mathrm{ccm}$ Plasma gelöst. Isoliert: 0,20 g Glykokollesterchlorhydrat (F. $144^{\circ}$ [korr.]) und 0,15g d-Alanin $\left([\alpha]_{\mathrm{D}}^{20}\right.$ des salzsauren Salzes $\left.=+10,0^{\circ}\right)$. An Anhydrid wurden 3 Fraktionen gewonnen. $[\alpha]_{D}^{20^{\circ}}$ von Fraktion 1 $(0,20 \mathrm{~g})=+4,0^{\circ}$, von Fraktion $2(0,15 \mathrm{~g})=+1,5^{0}$ und von Fraktion $3(0,5 \mathrm{~g})=0^{0}$.

4. dl-Alanyl-glycin: $2 \mathrm{~g}$ in $25 \mathrm{ccm}$ Plasma gelöst. Isoliert: $0,25 \mathrm{~g}$ Glykollesterchlorhydrat und $0,20 \mathrm{~g}$ d-Alanin. Drehung des salzsauren Salzes $=+9,0^{\circ}$. Aus dem Destillationsrückstand wurden $0,25 \mathrm{~g}$ Glycyl-l-alaninanhydrid von der spezifischen Drehung $+3,8^{\circ}$ isoliert. Eine weitere Fraktion von $0,8 \mathrm{~g}$ zeigte $[\alpha]_{\mathrm{D}}^{200}=+2,20$ und eine dritte Fraktion $(0,65 \mathrm{~g})$ war fast inaktiv.

5. Glycyl-dl-alanin: $2 \mathrm{~g}$ in $25 \mathrm{ccm}$ Plasma gelöst. Isoliert: $0,08 \mathrm{~g}$ Glykokollesterchlorhydrat und $0,1 \mathrm{~g}$ salzsaures Alanin. Es war etwas gefärbt. Seine Lösung drehte deutlich nach rechts, es war aber unmöglich, das Drehungsvermögen exakt zu bestimmen. An Anhydrid wurden gewonnen 1,25 g. Nur die erste Fraktion $(0,15 \mathrm{~g})$ war optisch-aktiv.

6. Glycyl-dl-alanin: $2 \mathrm{~g}$ in $25 \mathrm{ccm}$ Plasma gelöst. Isoliert: $0,05 \mathrm{~g}$ Glykokollesterchlorhydrat (F. $144^{\circ}$ [korr.]) und $0,08 \mathrm{~g}$ salzsaures Alanin. Es drehte nach rechts. $0,10 \mathrm{~g}$ Anhydrid drehten in wässeriger Lösung nach rechts, die übrigen $1,2 \mathrm{~g}$ waren optisch inaktiv.

7. Diglycyl-glycin: $2 \mathrm{~g}$ in $25 \mathrm{ccm}$ Plasma gelöst. Isoliert: $0,75 \mathrm{~g}$ Glykokollesterchlorhydrat und 0,52 $\mathrm{g}$ Glycinanhydrid.

8. Diglycyl-glycin: $1 \mathrm{~g}$ in $15 \mathrm{ccm}$ Plasma gelöst. Isoliert: $0,38 \mathrm{~g}$ Glykokollesterchlorhydrat und $0,20 \mathrm{~g}$ reines Glycinanhydrid. 